\title{
SOME CHARACTERIZATIONS OF HARDY SPACES ASSOCIATED WITH TWISTED CONVOLUTION
}

\author{
JIZHENG HUANG
}

(Received 6 August 2008)

\begin{abstract}
In this paper, we shall give some characterizations of the Hardy space associated with twisted convolution, including Lusin area integral, Littlewood-Paley $g$-function and heat maximal function.

2000 Mathematics subject classification: primary 42B30, 42B25, 42B35.

Keywords and phrases: twisted convolution, Hardy space, atomic decomposition, Lusin area integral, Littlewood-Paley $g$-function.
\end{abstract}

\section{Introduction}

On $\mathbb{C}^{n}$ consider the $2 n$ linear differential operators

$$
Z_{j}=\frac{\partial}{\partial_{z_{j}}}+\frac{1}{4} \overline{z_{j}}, \quad \overline{Z_{j}}=\frac{\partial}{\partial_{\overline{z_{j}}}}-\frac{1}{4} z_{j}, \quad j=1,2, \ldots, n .
$$

Together with the identity they generate a Lie algebra $h^{n}$ which is isomorphic to the $2 n+1$-dimensional Heisenberg algebra. The only nontrivial commutation relations are

$$
\left[Z_{j}, \overline{Z_{j}}\right]=-\frac{1}{2} I, \quad j=1,2, \ldots, n .
$$

The operator $L$ defined by

$$
L=-\frac{1}{2} \sum_{j=1}^{n}\left(Z_{j} \overline{Z_{j}}+\overline{Z_{j}} Z_{j}\right)
$$

is nonnegative, self-adjoint and elliptic. Therefore it generates a diffusion semigroup $\left\{T_{t}^{L}\right\}_{t>0}=\left\{e^{-t L}\right\}_{t>0}$. The operators in (1) generate a family of 'twisted translations'

The author was supported by the Specialized Research Fund for the Doctoral Program of Higher Education of China (No. 2007001040).

(C) 2009 Australian Mathematical Society 0004-9727/2009 \$16.00 
$\tau_{w}$ on $\mathbb{C}^{n}$ defined on measurable functions by

$$
\begin{aligned}
\left(\tau_{w} f\right)(z) & =\exp \left(\frac{1}{2} \sum_{j=1}^{n}\left(w_{j} z_{j}+\overline{w_{j} z_{j}}\right)\right) f(z) \\
& =f(z+w) \exp \left(\frac{i}{2} \operatorname{Im}(z \cdot \bar{w})\right) .
\end{aligned}
$$

The 'twisted convolution' of two functions $f$ and $g$ on $\mathbb{C}^{n}$ can now be defined as

$$
\begin{aligned}
(f \times g)(z) & =\int_{\mathbb{C}^{n}} f(w) \tau_{-w} g(z) d w \\
& =\int_{\mathbb{C}^{n}} f(z-w) g(w) \bar{\omega}(z, w) d w,
\end{aligned}
$$

where $\omega(z, w)=\exp (i \operatorname{Im}(z \cdot \bar{w}) / 2)$. More about twisted convolution can be found in $[1,7,9]$.

In [8] the authors defined the Hardy space $H_{L}^{1}\left(\mathbb{C}^{n}\right)$ associated with twisted convolution. They gave several characterizations of $H_{L}^{1}\left(\mathbb{C}^{n}\right)$, such as maximal function, atomic decomposition and Riesz transform. The purpose of this paper is to consider other characterizations, including Lusin area integral and LittlewoodPaley $g$-function. In order to prove our result, we also give a heat maximal functon characterization for $H_{L}^{1}\left(\mathbb{C}^{n}\right)$.

We first give some basic notation for $H_{L}^{1}\left(\mathbb{C}^{n}\right)$. Let $\mathcal{B}$ denote the class of $C^{\infty}$ functions $\varphi$ on $\mathbb{C}^{n}$, supported on the ball $B(0,1)$ such that $\|\varphi\|_{\infty} \leq 1$ and $\|\nabla \varphi\|_{\infty} \leq 2$. For $t>0$, let $\varphi_{t}(z)=t^{-2 n} \varphi(z / t)$. Given $\sigma>0, \quad 0<\sigma \leq+\infty$ and a tempered distribution $f$, define the grand maximal function

$$
M_{\sigma} f(z)=\sup _{\varphi \in \mathcal{B}} \sup _{0<t<\sigma}\left|\varphi_{t} \times f(z)\right| .
$$

Then the Hardy space $H_{L}^{1}\left(\mathbb{C}^{n}\right)$ can be defined by

$$
H_{L}^{1}\left(\mathbb{C}^{n}\right)=\left\{f \in L^{1}\left(\mathbb{C}^{n}\right) \mid M_{\infty} f \in L^{1}\left(\mathbb{C}^{n}\right)\right\} .
$$

We define atoms for $H_{L}^{1}\left(\mathbb{C}^{n}\right)$ as follows. A function $a(z)$ is an atom for the Hardy space $H_{L}^{1}\left(\mathbb{C}^{n}\right)$ associated to a ball $B\left(z_{0}, r\right)$ if the following properties hold.

(1) supp $a \subset B\left(z_{0}, r\right)$.

(2) $\|a\|_{\infty} \leq\left|B\left(z_{0}, r\right)\right|^{-1}$.

(3) $\int a(w) \bar{\omega}\left(z_{0}, w\right) d w=0$.

The atomic quasi-norm in $H_{L}^{1}\left(\mathbb{C}^{n}\right)$ is defined by

$$
\|f\|_{L \text {-atom }}=\inf \left\{\Sigma\left|\lambda_{j}\right|\right\}
$$

where the infimum is taken over all decompositions $f=\sum \lambda_{j} a_{j}$ and $a_{j}$ are atoms.

The following result has been proved in [8]. 
PROPOSITION 1. For a tempered distribution $f$ on $\mathbb{C}^{n}$ the following properties are equivalent.

(i) $M_{\infty} f \in L^{1}\left(\mathbb{C}^{n}\right)$.

(ii) For some $\sigma, 0<\sigma<+\infty, M_{\sigma} f \in L^{1}\left(\mathbb{C}^{n}\right)$.

(iii) For some radial $\varphi \in \mathcal{S}$, such that $\int \varphi(z) d z \neq 0$, we have

$$
\sup _{0<t<1}\left|\varphi_{t} \times f(z)\right| \in L^{1}\left(\mathbb{C}^{n}\right) .
$$

(iv) The distribution $f$ can be decomposed as $f=\sum \lambda_{j} a_{j}$, where $a_{j}$ are atoms and $\sum\left|\lambda_{j}\right|<+\infty$.

It is well known that there are many equivalent characterizations of the classical Hardy spaces (see [4]), we shall consider other characterizations for $H_{L}^{1}\left(\mathbb{C}^{n}\right)$ in this paper.

We define the Lusin area integral operator by

$$
\left(S_{L}^{\alpha} f\right)(z)=\left(\int_{0}^{+\infty} \int_{|z-w|<\alpha t}\left|Q_{t}^{L} f(w)\right|^{2} \frac{d w d t}{t^{2 n+1}}\right)^{1 / 2}
$$

where $Q_{t}^{L} f(x)=t^{2}\left(\left.\partial_{s} T_{s}^{L}\right|_{s=t^{2}} f\right)(z)$.

REMARK 2. It is easy to see that the definition of area integral operator is independent of $\alpha$ in the sense of $\left\|\left(S_{L}^{\alpha} f\right)\right\|_{L^{p}} \sim\left\|\left(S_{L}^{\beta} f\right)\right\|_{L^{p}}$, for $0<\alpha<\beta<\infty$ and $0<p<\infty$ (see [3]). In the following we use $S_{L}$ to denote $S_{L}^{1}$.

We can characterize $H_{L}^{1}\left(\mathbb{C}^{n}\right)$ as follows.

THEOREM 3. A function $f \in H_{L}^{1}\left(\mathbb{C}^{n}\right)$ if and only if its area integral $S_{L} f \in L^{1}\left(\mathbb{C}^{n}\right)$ and $f \in L^{1}\left(\mathbb{C}^{n}\right)$. Moreover,

$$
\|f\|_{H_{L}^{1}} \sim\left\|S_{L} f\right\|_{L^{1}}
$$

The Littlewood-Paley $g$-function is defined by

$$
\mathcal{G}_{L}(f)(z)=\left(\int_{0}^{\infty}\left|Q_{t}^{L} f(z)\right|^{2} \frac{d t}{t}\right)^{1 / 2} .
$$

The Hardy space $H_{L}^{1}\left(\mathbb{C}^{n}\right)$ can also be characterized by $\mathcal{G}_{L}$ as in the following theorem.

TheOREM 4. A function $f \in H_{L}^{1}\left(\mathbb{C}^{n}\right)$ if and only if $\mathcal{G}_{L} f \in L^{1}\left(\mathbb{C}^{n}\right)$ and $f \in L^{1}\left(\mathbb{C}^{n}\right)$. Moreover,

$$
\|f\|_{H_{L}^{1}} \sim\left\|\mathcal{G}_{L} f\right\|_{L^{1}}
$$

We also need some basic propositions about the tent space (see [3]). 
Let $0<p<\infty$, and $1 \leq q \leq \infty$, then the tent space $T_{q}^{p}$ is defined as the space of functions $f$ on $\mathbb{C}^{n} \times \mathbb{R}^{+}$, so that

$$
\left(\int_{\Gamma(z)}|f(w, t)|^{q} \frac{d w d t}{t^{2 n+1}}\right)^{1 / q} \in L^{p}\left(\mathbb{C}^{n}\right) \quad \text { when } 1 \leq q<\infty
$$

and

$$
\sup _{(w, t) \in \Gamma(z)}|f(w, t)| \in L^{p}\left(\mathbb{C}^{n}\right) \quad \text { when } q=\infty,
$$

where $\Gamma(z)$ is the standard cone whose vertex is $z \in \mathbb{C}^{n}$, that is,

$$
\Gamma(z)=\{(w, t):|w-z|<t\}
$$

Assume $B\left(z_{0}, r\right)$ is a ball in $\mathbb{C}^{n}$, its tent $\widehat{B}$ is defined by

$$
\widehat{B}=\left\{(w, t):\left|w-z_{0}\right| \leq r-t\right\} .
$$

A function $a(z, t)$ that supported in a tent $\widehat{B}, B$ is a ball in $\mathbb{C}^{n}$, is said to be an atom in the tent space $T_{q}^{p}$ if and only if it satisfies

$$
\left(\int_{\widehat{B}}|a(z, t)|^{2} \frac{d z d t}{t}\right)^{1 / 2} \leq|B|^{1 / 2-1 / p} .
$$

The atomic decomposition of $T_{q}^{p}$ is stated as in the following proposition.

PROPOSITION 5. When $0<p \leq 1$, then any $f \in T_{2}^{p}$ can be written as $f=\sum \lambda_{k} a_{k}$, where $a_{k}$ are atoms and $\sum\left|\lambda_{k}\right|^{p} \leq C\|f\|_{T_{2}^{p}}^{p}$.

The paper is organized as follows: in Section 2, we give some estimates of the kernels; in Section 3, we prove the main results of this paper.

Throughout the article, we shall use $A$ and $C$ to denote the positive constants, which are independent of main parameters and may be different at each occurrence. By $B_{1} \sim B_{2}$, we mean that there exists a constant $C>1$ such that $1 / C \leq B_{1} / B_{2} \leq C$.

\section{Preliminaries}

In this section, we give some estimates of the kernel of $Q_{t}^{L}$ that we shall use subsequently.

Let $\left\{T_{t}^{L}\right\}_{t>0}$ be the heat semigroup generated by the operator $L$, then, for $f \in$ $L^{2}\left(\mathbb{C}^{n}\right)$, the function $e^{-t L} f$ has the special Hermite expansion (see [11])

$$
e^{-t L} f(z)=(2 \pi)^{-n} \sum_{k=0}^{\infty} e^{-(2 k+n) t} f \times \varphi_{k}(z),
$$


where $\varphi_{k}$ is the Laguerre function. Therefore $e^{-t L} f$ is given by twisted convolution with the kernel

$$
K_{t}(z)=(2 \pi)^{-n} \sum_{k=0}^{\infty} e^{-(2 k+n) t} \varphi_{k}(z) .
$$

Let

$$
L_{k}^{\alpha}(x)=\sum_{j=0}^{k} \frac{\Gamma(k+\alpha+1)}{\Gamma(k-j+1) \Gamma(j+\alpha+1)} \frac{(-x)^{j}}{j !}
$$

be the Laguerre polynomials of type $\alpha$ and degree $k$, then we have the following generating function for Laguerre polynomials:

$$
\sum_{k=0}^{\infty} L_{k}^{\alpha}(x) r^{k}=(1-r)^{-\alpha-1} e^{-(r /(1-r)) x} .
$$

From (4) we obtain

$$
K_{t}(z)=(4 \pi)^{-n}(\sinh t)^{-n} e^{-\frac{1}{4}|z|^{2}(\operatorname{coth} t)} .
$$

It is easy to prove that the heat kernel $K_{t}(z)$ has the following estimates (see the proof of Lemma 7).

LEMMA 6. There exists a positive constant $C>0$ such that the following inequalities hold.

(i) $\left|K_{t}(z)\right| \leq C t^{-n} e^{-C|z|^{2} / t}$.

(ii) $\left|\nabla K_{t}(z)\right| \leq C t^{-n-\frac{1}{2}} e^{-C|z|^{2} / t}$.

Let $Q_{t}^{L}(z)$ be the twisted convolution kernel of $Q_{t}^{L}$, then

$$
Q_{t}^{L}(z)=\left.t^{2} \partial_{s} K_{s}(z)\right|_{s=t^{2}} .
$$

LEMMA 7. There exists a constant $C>0$ such that the following inequalities hold.

(i) $\left|Q_{t}^{L}(z)\right| \leq C t^{-2 n} e^{-C t^{-2}|z|^{2}}$.

(ii) $\left|\nabla Q_{t}^{L}(z)\right| \leq C t^{-2 n-1} e^{-C t^{-2}|z|^{2}}$.

PROOF. It is easy to see that

$$
\begin{aligned}
\partial_{t} K_{t}(z)= & (4 \pi)^{-n}(-n)(\sinh t)^{-n-1}(\cosh t) e^{-\frac{1}{4}|z|^{2}(\operatorname{coth} t)} \\
& +(4 \pi)^{-n}(\sinh t)^{-n-2}\left(-\frac{1}{4}|z|^{2}\right) e^{-\frac{1}{4}|z|^{2}(\operatorname{coth} t)}
\end{aligned}
$$

Therefore

$$
\begin{aligned}
\left|\partial_{t} K_{t}(z)\right| \leq & C\left((\sinh t)^{-n-1}(\cosh t) e^{-\frac{1}{4}|z|^{2}(\operatorname{coth} t)}\right. \\
& \left.+(\sinh t)^{-n-2}|z|^{2} e^{-\frac{1}{4}|z|^{2}(\operatorname{coth} t)}\right)
\end{aligned}
$$


Noting that there exists $C_{1}>0$ such that for all $t>0$ we have

$$
\sinh t \geq C_{1} t, \quad \operatorname{coth} t \geq C_{1} t^{-1}
$$

and

$$
(\sinh t)^{-n-1} \cosh t \leq \begin{cases}C_{1}(\sinh t)^{-n-1} \leq C_{1} t^{-n-1} & \text { if } 0<t \leq 1 \\ C_{1}(\sinh t)^{-n} \operatorname{coth} t \leq C_{1} t^{-n-1} & \text { if } 1<t<\infty\end{cases}
$$

From (6) and (7) we have

$$
\begin{aligned}
\left|\partial_{t} K_{t}(z)\right| & \leq C\left(t^{-n-1} e^{-\frac{1}{4} t^{-1}|z|^{2}}+t^{-n-2}|z|^{2} e^{-\frac{1}{4} t^{-1}|z|^{2}}\right) \\
& \leq C t^{-n-1} e^{-C t^{-1}|z|^{2}} .
\end{aligned}
$$

So, we have

$$
\left|Q_{t}^{L}(z)\right| \leq C t^{-2 n} e^{-C t^{-2}|z|^{2}}
$$

This proves part (i).

To prove part (ii), it is sufficient to prove

$$
\left|\partial_{z_{j}} \partial_{t} K_{t}(z)\right| \leq C t^{-n-\frac{3}{2}} e^{-C t^{-1}|z|^{2}}, \quad j=1,2, \ldots, n .
$$

It is easy to calculate

$$
\begin{aligned}
\partial_{z_{j}} \partial_{t} K_{t}(z)= & (4 \pi)^{-n}(-n)(\sinh t)^{-n-1}(\cosh t) 2 z_{j}\left(-\frac{1}{4} \operatorname{coth} t\right) e^{-\frac{1}{4}|z|^{2}(\operatorname{coth} t)} \\
+ & (4 \pi)^{-n}(\sinh t)^{-n-2}\left(-\frac{1}{4} \cosh t\right) 2 z_{j}\left(-\frac{1}{4} \operatorname{coth} t\right) \\
& \times\left(-\frac{1}{4}|z|^{2}\right) e^{-\frac{1}{4}|z|^{2}(\operatorname{coth} t)} \\
& +(4 \pi)^{-n}(\sinh t)^{-n-2}\left(-\frac{1}{2} z_{j}\right) e^{-\frac{1}{4}|z|^{2}(\operatorname{coth} t)} .
\end{aligned}
$$

By (6) and (7),

$$
\begin{aligned}
\left|\partial_{z_{j}} \partial_{t} K_{t}(z)\right| \leq & C\left(t^{-n-\frac{3}{2}} \frac{|z|}{\sqrt{t}} e^{-\frac{1}{4} t^{-1}|z|^{2}}+t^{-n-\frac{3}{2}}\left(\frac{|z|}{\sqrt{t}}\right)^{3} e^{-\frac{1}{4} t^{-1}|z|^{2}}\right. \\
& \left.+t^{-n-\frac{3}{2}} \frac{|z|}{\sqrt{t}} e^{-\frac{1}{4} t^{-1}|z|^{2}}\right) \\
\leq & C t^{-n-\frac{3}{2}} e^{-C t^{-1}|z|^{2}}
\end{aligned}
$$

This completes the proof of (8) and so part (ii) is proved.

We can also consider the following operator $Q_{t}^{2}=\left.t^{4} \partial_{s}^{2} T_{s}^{L}\right|_{s=t^{2}}$. If we use $Q_{t}^{2}(z)$ to denote the twisted convolution kernel of $Q_{t}^{2}$, then similarly as Lemma 7, we can prove the following result.

LEMMA 8. There exists a constant $C>0$ such that the following inequalities hold.

(i) $\left|Q_{t}^{2}(z)\right| \leq C t^{-2 n} e^{-C t^{-2}|z|^{2}}$.

(ii) $\left|\nabla Q_{t}^{2}(z)\right| \leq C t^{-2 n-1} e^{-C t^{-2}|z|^{2}}$. 


\section{The proofs of the main results}

In this section, we shall give the proofs of the main results of this paper.

We first give the maximal function characterization for $H_{L}^{1}\left(\mathbb{C}^{n}\right)$. By Proposition 1 , we have the following lemma (see [8, p. 281]).

LEMMA 9. $f \in H_{L}^{1}\left(\mathbb{C}^{n}\right)$ if and only if

$$
\tilde{M} f(z)=\sup _{0<t<1}\left|K_{t} \times f(z)\right| \in L^{1}\left(\mathbb{C}^{n}\right)
$$

and $f \in L^{1}\left(\mathbb{C}^{n}\right)$.

Let

$$
M f(z)=\sup _{t>0}\left|K_{t} \times f(z)\right| \in L^{1}\left(\mathbb{C}^{n}\right),
$$

then we can characterize $H_{L}^{1}\left(\mathbb{C}^{n}\right)$ by the maximal function $M f$ as follows.

THEOREM 10. $f \in H_{L}^{1}\left(\mathbb{C}^{n}\right)$ if and only if $M f \in L^{1}\left(\mathbb{C}^{n}\right)$ and $f \in L^{1}\left(\mathbb{C}^{n}\right)$.

Proof. By Lemma 9, we know $f \in H_{L}^{1}\left(\mathbb{C}^{n}\right)$ if $M f \in L^{1}\left(\mathbb{C}^{n}\right)$ and $f \in L^{1}\left(\mathbb{C}^{n}\right)$.

For the reverse, we just need to prove that there exists $C>0$ such that for any atom $a(z)$ of $H_{L}^{1}\left(\mathbb{C}^{n}\right)$,

$$
\|M a\|_{L^{1}} \leq C .
$$

Let $a(z)$ be an atom of $H_{L}^{1}\left(\mathbb{C}^{n}\right)$. By twisted translation, we can assume that supp $a \subset$ $B(0, r)$. Then, we have $\int a(w) d w=0$.

By Lemma 6, we have

$$
\int_{|w| \leq 2 r}|M a(w)| d w \leq\left\|M_{H-L} a\right\|_{L^{2}}(2 r)^{n} \leq C,
$$

where $M_{H-L}$ is the Hardy-Littlewood maximal function.

For $|z|>2 r$, we get

$$
\begin{aligned}
K_{t} \times a(z) & =\int K_{t}(z-w) a(w) \bar{\omega}(z, w) d w \\
& =\int\left(K_{t}(z-w)-K_{t}(z)\right) a(w) \bar{\omega}(z, w) d w+K_{t}(z) \widehat{a}\left(-\frac{i}{2} z\right) \\
& =I_{1}+I_{2} .
\end{aligned}
$$

By Lemma 6 again, we can prove

$$
I_{1} \leq C \int t^{-n-\frac{1}{2}} e^{-C t^{-1}|z-w|^{2}}|w||a(w)| d w \leq C r|z|^{-2 n-1} .
$$

Therefore

$$
\int_{|z|>2 r}\left|I_{1}\right| d z \leq C
$$


By Lemma 6 (i) and Hardy's inequality (see [6, p. 341, Theorem 7.22]), we get

$$
\int_{|z|>2 r}\left|I_{2}\right| d z \leq C \int_{\mathbb{C}^{n}} \frac{|\widehat{a}(-(i / 2) z)|}{|z|^{2 n}} d z \leq C .
$$

Therefore we have $\|M a\|_{L^{1}} \leq C$ and Theorem 10 is proved.

In order to get our results, we need the following lemma.

LEMMA 11. The operators $S_{L}$ and $\mathcal{G}_{L}$ are isometries on $L^{2}\left(\mathbb{C}^{n}\right)$ up to constant factors. Exactly,

$$
\left\|\mathcal{G}_{L} f\right\|_{L^{2}}=\frac{1}{2}\|f\|_{L^{2}}, \quad\left\|S_{L} f\right\|_{L^{2}}=\frac{\sqrt{c_{n}}}{2}\|f\|_{L^{2}} .
$$

Proof. The $L^{2}$ equality for $\mathcal{G}_{L}$ is established in [12, Proposition 3.1]. As a consequence we have

$$
\begin{aligned}
\left\|S_{L} f\right\|_{L^{2}}^{2} & =\int_{\mathbb{C}^{n}} \int_{\mathbb{C}^{n} \times \mathbb{R}^{+}}\left|Q_{t}^{L} f(w)\right|^{2} \chi_{\Gamma(z)}(w, t) \frac{d w d t}{t^{2 n+1}} d z \\
& =c_{n} \int_{\mathbb{C}^{n} \times \mathbb{R}^{+}}\left|Q_{t}^{L} f(w)\right|^{2} \frac{d w d t}{t}=c_{n}\left\|\mathcal{G}_{L} f\right\|_{L^{2}}^{2} \\
& =\frac{c_{n}}{4}\|f\|_{L^{2}}^{2} .
\end{aligned}
$$

Proof of TheOREM 3. As in the proof of Theorem 10, by Lemma 7, we can prove that there exists a constant $C>0$ such that for any atom $a(z)$ of $H_{L}^{1}\left(\mathbb{C}^{n}\right)$ we have

$$
\left\|S_{L} a\right\|_{L^{1}} \leq C
$$

Now we prove that $f \in H_{L}^{1}\left(\mathbb{C}^{n}\right)$ when $S_{L} f \in L^{1}\left(\mathbb{C}^{n}\right)$ and $f \in L^{1}\left(\mathbb{C}^{n}\right)$.

We first assume that $f \in L^{1}\left(\mathbb{C}^{n}\right) \cap L^{2}\left(\mathbb{C}^{n}\right)$. When $S_{L} f \in L^{1}\left(\mathbb{C}^{n}\right)$, we know that $Q_{t}^{L} f \in T_{2}^{1}$. By Proposition 5 we get

$$
Q_{t}^{L} f(z)=\sum_{j} \lambda_{j} a_{j}(z, t)
$$

where $a_{j}(z, t)$ are atoms of $T_{2}^{1}$ and $\sum_{j}\left|\lambda_{j}\right|<\infty$. By the spectral theorem (see [10]), we can prove

$$
f(z)=4 \int_{0}^{\infty} Q_{t}^{L}\left(Q_{t}^{L} f(z)\right) \frac{d t}{t}
$$

By (10) and (11), we get

$$
f(z)=4 \int_{0}^{+\infty} Q_{t}^{L}\left(\sum_{j} \lambda_{j} a_{j}(z, t)\right) \frac{d t}{t}=C \sum_{j} \lambda_{j} \int_{0}^{+\infty} Q_{t}^{L} a_{j}(z, t) \frac{d t}{t} .
$$


Therefore it is sufficient to prove that the functions

$$
\alpha_{j}=\int_{0}^{+\infty} Q_{t}^{L} a_{j}(z, t) \frac{d t}{t}, \quad i=1,2, \ldots,
$$

are bounded in $H_{L}^{1}\left(\mathbb{C}^{n}\right)$ uniformly; that is, there exists a constant $C>0$ such that for any atom $a(z, t)$ in $T_{2}^{1}$,

$$
\|\alpha\|_{H_{L}^{1}}=\left\|\int_{0}^{+\infty} Q_{t}^{L} a(z, t) \frac{d t}{t}\right\|_{H_{L}^{1}} \leq C .
$$

We assume that $a(z, t)$ is supported in $\widehat{B}\left(z_{0}, r\right)$, where $\widehat{B}\left(z_{0}, r\right)$ denotes the tent of the ball $B\left(z_{0}, r\right)$, then

$$
\begin{aligned}
& \left\|\sup _{t>0}\left|e^{-t L} \alpha(z)\right|\right\|_{L^{1}} \leq\left\|\left(\sup _{t>0}\left|e^{-t L} \alpha(z)\right|\right) \chi_{B^{*}}\right\|_{L^{1}}+\left\|\left(\sup _{t>0}\left|e^{-t L} \alpha(z)\right|\right) \chi_{\left(B^{*}\right)^{c}}\right\|_{L^{1}} \\
& =I_{1}+I_{2} \text {, }
\end{aligned}
$$

where $B^{*}=B\left(z_{0}, 2 r\right)$.

By the Hölder inequality, we get

$$
I_{1} \leq\left|B^{*}\right|^{1 / 2}\left(\int_{\mathbb{C}^{n}}\left(\sup _{t>0}\left|e^{-t L} \alpha(z)\right|\right)^{2} d z\right)^{1 / 2} \leq\left|B^{*}\right|^{1 / 2}\|\alpha\|_{L^{2}} .
$$

By the self-adjointness of $Q_{t}^{L}$ and Lemma 11 we have

$$
\begin{aligned}
\|\alpha\|_{L^{2}} & =\sup _{\|\beta\|_{L^{2} \leq 1}} \int_{\mathbb{C}^{n}} \alpha(z) \bar{\beta}(z) d z \\
& =\sup _{\|\beta\|_{L^{2} \leq 1}} \int_{\mathbb{C}^{n}}\left(\int_{0}^{+\infty} Q_{t}^{L} a(z, t) \frac{d t}{t}\right) \bar{\beta}(z) d z \\
& =\sup _{\|\beta\|_{L^{2} \leq 1}} \int_{0}^{+\infty} \int_{\mathbb{C}^{n}} Q_{t}^{L} a(z, t) \bar{\beta}(z) d z \frac{d t}{t} \\
& =\sup _{\|\beta\|_{L^{2}} \leq 1} \int_{0}^{+\infty} \int_{\mathbb{C}^{n}} a(z, t) Q_{t}^{L} \bar{\beta}(z) d z \frac{d t}{t} \\
& \leq \sup _{\|\beta\|_{L^{2}} \leq 1}\left(\int_{\mathbb{C}^{n}} \int_{0}^{+\infty}|a(z, t)|^{2} \frac{d z d t}{t}\right)^{1 / 2} \\
& \times\left(\int_{\mathbb{C}^{n}} \int_{0}^{+\infty}\left|Q_{t}^{L} \bar{\beta}(z)\right|^{2} \frac{d z d t}{t}\right)^{1 / 2} \\
& \leq|B|^{-1 / 2}\|\beta\|_{L^{2}} \leq|B|^{-1 / 2} .
\end{aligned}
$$

This proves $I_{1} \leq C$. 
By Lemma 7 we can prove

$$
\begin{aligned}
& \sup _{s>0}\left|e^{-s L} \int_{0}^{+\infty} Q_{t}^{L} a(z, t) \frac{d t}{t}\right| \\
& =\sup _{s>0}\left|e^{-s L} \int_{0}^{+\infty}(-L) t e^{-t L} a(z, t) \frac{d t}{t}\right| \\
& =\sup _{s>0}\left|\int_{0}^{+\infty}(-t L) e^{-(s+t) L} a(z, t) \frac{d t}{t}\right| \\
& =\sup _{s>0}\left|\int_{0}^{+\infty}\left(\frac{t}{s+t}\right)((s+t) L) e^{-(s+t) L} a(z, t) \frac{d t}{t}\right| \\
& =\sup _{s>0}\left|\int_{0}^{+\infty}\left(\frac{t}{s+t}\right) \int_{\mathbb{C}^{n}} Q_{s+t}^{L}(z, w) a(w, t) \frac{d w d t}{t}\right| \\
& \leq \sup _{s>0} \int_{0}^{+\infty} \frac{t}{s+t} \int_{\mathbb{C}^{n}}(s+t)^{-2 n} \exp \left(-\frac{|z-w|^{2}}{(s+t)^{2}}\right)|a(w, t)| \frac{d w d t}{t} \\
& \leq \sup _{s>0} \int_{0}^{+\infty} \frac{t}{s+t} \int_{\mathbb{C}^{n}}(s+t)^{-2 n}\left(1+\frac{|z-w|^{2}}{(s+t)^{2}}\right)^{-(n+1)}|a(w, t)| \frac{d w d t}{t} \\
& \leq \sup _{s>0}\left(\int_{0}^{r} \int_{B}(s+t)^{-4 n}\left(1+\frac{|z-w|^{2}}{(s+t)^{2}}\right)^{-(2 n+1)}\left(\frac{t}{s+t}\right)^{2} \frac{d w d t}{t}\right)^{1 / 2} \\
& \times\left(\int_{0}^{r} \int_{B}|a(w, t)|^{2} \frac{d w d t}{t}\right)^{1 / 2} \\
& \leq|B|^{-1 / 2}\left|z-z_{0}\right|^{2 n+1}\left(\int_{0}^{r} \int_{B} t d w d t\right)^{1 / 2} \\
& \leq C r\left|z-z_{0}\right|^{-(2 n+1)} \text {. }
\end{aligned}
$$

Then we have

$$
I_{2} \leq C r \int_{\left(B^{*}\right)^{c}}\left|z-z_{0}\right|^{-(2 n+1)} d z \leq C .
$$

When $f \in L^{1}\left(\mathbb{C}^{n}\right)$ we can prove the result as in [2, Proposition 14]. In fact, we let $f_{k}=T_{2^{-k}}^{L} f, \quad k \geq 0$. Then, by $f \in L^{1}\left(\mathbb{C}^{n}\right)$ and Lemma 6, we know $f_{k} \in L^{2}\left(\mathbb{C}^{n}\right)$ and $\left\|S_{L} f_{k}\right\|_{1} \leq\left\|S_{L} f\right\|$. By the case of $f \in L^{1} \cap L^{2}$, we get

$$
\left\|f_{k}\right\|_{H_{L}^{1}\left(\mathbb{C}^{n}\right)} \lesssim\left\|S_{L} f_{k}\right\|_{L^{1}} \leq\left\|S_{L} f\right\|_{L^{1}}
$$

By the monotone theorem, we have

$$
\left\|f_{k}-f_{n}\right\|_{H_{L}^{1}} \leq\left\|S_{k}^{L}\left(f_{k}-f_{n}\right)\right\|_{L^{1}} \rightarrow 0 \quad \text { when } k, n \rightarrow+\infty .
$$

Therefore $\left\{f_{k}\right\}$ is a Cauchy sequence in $H_{L}^{1}\left(\mathbb{R}^{d}\right)$ and there exists $g \in H_{L}^{1}\left(\mathbb{R}^{d}\right)$ such that

$$
\lim _{m \rightarrow+\infty} f_{k}=g \quad \text { in } H_{L}^{1}\left(\mathbb{R}^{d}\right) \text {. }
$$


As

$$
\lim _{k \rightarrow+\infty} f_{k}=f \quad \text { in }\left(B M O_{L}\right)^{*},
$$

we know $f=g \in H_{L}^{1}\left(\mathbb{R}^{d}\right)$ and $\|f\|_{H_{L}^{1}\left(\mathbb{C}^{n}\right)} \lesssim\left\|S_{L} f\right\|_{L^{1}}$.

This gives the proof of Theorem 3 .

We define $\widetilde{S_{L}^{\alpha}} f(z)$ by

$$
\widetilde{S_{L}}(f)(z)=\left(\int_{0}^{+\infty} \int_{|z-w|<\alpha t}\left|Q_{t}^{2} f(w)\right|^{2} \frac{d w d t}{t^{2 n+1}}\right)^{1 / 2}
$$

where $\alpha>0$. Then in the same way as the proof of Theorem 3, we can prove the following result.

COROllary 12. A function $f \in H_{L}^{1}\left(\mathbb{C}^{n}\right)$ if and only if its area integral $\widetilde{S_{L}^{\alpha}} f \in$ $L^{1}\left(\mathbb{C}^{n}\right)$ and $f \in L^{1}\left(\mathbb{C}^{n}\right)$. Moreover,

$$
\|f\|_{H_{L}^{1}} \sim\left\|\widetilde{S_{L}^{\alpha}} f\right\|_{L^{1}}
$$

Now we can give the proof of Theorem 4.

Proof of TheOrem 4. Firstly, by Lemma 7, we can prove that there exists a positive constant $C$ such that for any atom $a(z)$ of $H_{L}^{1}\left(\mathbb{C}^{n}\right)$, we have

$$
\left\|\mathcal{G}_{L} a\right\|_{L^{1}} \leq C .
$$

For the converse, by Corollary 12, it is sufficient to prove

$$
\left\|\widetilde{S_{L}^{1}} f\right\|_{L^{1}} \leq C\left\|\mathcal{G}_{L} f\right\|_{L^{1}}
$$

Our proof is motivated by [5]. Let

$$
F(z)(t)=\left(\partial_{t} e^{-t L} f\right)(z), \quad V(z, s)=e^{-s L} F(z),
$$

then

$$
V(z, s)(t)=e^{-s L}\left(\partial_{t} e^{-t L} f\right)(z)=\left(\partial_{t} e^{-(s+t) L} f\right)(z)
$$

Therefore

$$
\begin{aligned}
\int_{0}^{+\infty}|V(z, s)(t)|^{2} t d t & =\int_{0}^{+\infty}\left|\left(\partial_{t} e^{-(s+t) L} f\right)(z)\right|^{2} t d t \\
& =\int_{s}^{+\infty}\left|\left(\partial_{t} e^{-t L} f\right)(z)\right|^{2}(t-s) d t
\end{aligned}
$$

Hence

$$
\sup _{s>0} \int_{0}^{+\infty}|V(z, s)(t)|^{2} t d t \leq \int_{0}^{+\infty}\left|\left(\partial_{t} e^{-t L} t f\right)(z)\right|^{2} t d t=\left(\mathcal{G}_{L} f(z)\right)^{2} .
$$


Let $\mathbf{X}=L^{2}((0, \infty), t d t)$, then

$$
\sup _{s>0}\left\|e^{-s L} F(z)\right\|_{\mathbf{X}}=\mathcal{G}_{L} f(z) \in L^{1}\left(\mathbb{C}^{n}\right) .
$$

Therefore $F \in H_{\mathbf{X}}^{1}\left(\mathbb{C}^{n}\right)$ and here $H_{\mathbf{X}}^{1}\left(\mathbb{C}^{n}\right)$ can be seen as a vector-valued Hardy space. This shows that $S_{1}^{2} F(z) \in L^{1}\left(\mathbb{C}^{n}\right)$, where

$$
S_{1}^{2} F(z)=\left(\int_{0}^{+\infty} \int_{|z-w|<2 t}\left\|Q_{t}^{L} F(w)\right\|_{\mathbf{X}}^{2} \frac{d w d t}{t^{2 n+1}}\right)^{1 / 2} .
$$

By

$$
\begin{aligned}
\left(S_{1}^{2} F(z)\right)^{2} & =\int_{0}^{+\infty} \int_{|z-w|<2 t}\left\|Q_{t}^{L} F(z)\right\|_{\mathbf{X}}^{2} \frac{d w d t}{t^{2 n+1}} \\
& =\int_{0}^{+\infty} \int_{|z-w|<2 t} \int_{0}^{+\infty}\left|t(-L) e^{-t L} F(w)(s)\right|^{2} s d s \frac{d w d t}{t^{2 n+1}} \\
& =\int_{0}^{+\infty} \int_{0}^{+\infty} \int_{|z-w|<2 t}\left|(-L)^{2} e^{-(s+t) L} f(w)\right|^{2} t^{1-2 n} s d w d t d s \\
& =\int_{0}^{+\infty} \int_{s}^{+\infty} \int_{|z-w|<2(t-s)}\left|(-L)^{2} e^{-t L} f(w)\right|^{2}(t-s)^{1-2 n} s d w d t d s \\
& =\int_{0}^{+\infty} \int_{0}^{t} \int_{|z-w|<2(t-s)}\left|(-L)^{2} e^{-t L} f(w)\right|^{2}(t-s)^{1-2 n} s d w d s d t \\
& \geq \int_{0}^{+\infty} \int_{0}^{t / 2} \int_{|z-w|<2(t-s)}\left|(-L)^{2} e^{-t L} f(w)\right|^{2}(t-s)^{1-2 n} s d w d s d t \\
& \geq \int_{0}^{+\infty} \int_{0}^{t / 2} \int_{|z-w|<t}\left|(-L)^{2} e^{-t L} f(w)\right|^{2} t^{1-2 n} s d w d s d t \\
& =\frac{1}{8} \int_{0}^{+\infty} \int_{|z-w|<t}\left|(-L)^{2} e^{-t L} f(w)\right|^{2} t^{3-2 n} d w d t \\
& =\frac{1}{8} \int_{0}^{+\infty} \int_{|z-w|<t}\left|Q_{t}^{2} f(w)\right|^{2} \frac{d w d t}{t^{2 n+1}}=\frac{1}{8}\left(\widetilde{S}_{L}^{1} f(z)\right)^{2},
\end{aligned}
$$

we get $\widetilde{S_{L}^{1}} f \in L^{1}\left(\mathbb{C}^{n}\right)$. Then $f \in H_{L}^{1}\left(\mathbb{C}^{n}\right)$ follows from Corollary 12

This completes the proof of Theorem 4.

\section{References}

[1] R. F. Anderson, 'The multiplicative Weyl functional calculus', J. Funct. Anal. 9 (1972), 423-440.

[2] P. Auscher and E. Russ, 'Hardy spaces and divergence operators on strongly Lipschitz domains of $\mathbb{R}^{n}$, J. Funct. Anal. 201 (2003), 148-184.

[3] R. R. Coifman, Y. Meyer and E. M. Stein, 'Some new function spaces and their applications to harmonic analysis', J. Funct. Anal. 62 (1985), 304-335. 
[4] C. Fefferman and E. M. Stein, ' $H^{p}$ spaces of several variables', Acta. Math. 129 (1972), 137-193.

[5] G. B. Folland and E. M. Stein, Hardy Spaces on Homogeneous Groups (Princeton University Press, Princeton, NJ, 1982).

[6] J. Garcia-Cuerva and J. L. Rubio de Francia, Weighted Norm Inequalities and Related Topics, North-Holland Mathematics Studies, 116 (North-Holland, Amsterdam, 1985).

[7] A. Grassman, G. Loupias and E. M. Stein, 'An algebra of pseudo-differential operators and quantum mechanics in phase space', Ann. Inst. Fourier (Grenoble) 18 (1969), 343-368.

[8] G. Mauceri, M. Picardello and F. Ricci, 'A Hardy space associated with twisted convolution', $A d v$. Math. 39 (1981), 270-288.

[9] J. Peetre, 'The Weyl transform and Laguerre polynomials', Le Matematiche 27 (1972), 301-323.

[10] E. M. Stein, Topics in Harmonic Analysis Related to the Littlewood-Paley Theory (Princeton University Press, Princeton, NJ, 1970).

[11] S. Thangavelu, Lectures on Hermite and Laguerre Expansions, Math. Notes, 42 (Princeton University Press, Princeton, NJ, 1993).

[12] _ _ 'Littlewood-Paley-Stein theory on $\mathbb{C}^{n}$ and Weyl multipliers', Rev. Mat. Iberoamericana 6 (1990), 75-90.

JIZHENG HUANG, CIAS, China Economics and Management Academy, Central University of Finance and Economics, Beijing, 100081, PR China e-mail: hjzheng@163.com 